\title{
RHIC Low Energy Beam Loss Projections
}

\author{
T. Satogata
}

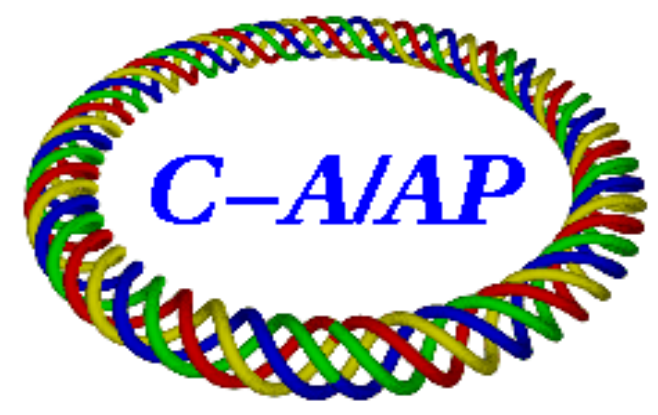

\section{Collider-Accelerator Department Brookhaven National Laboratory Upton, NY 11973}

Notice: This document has been authorized by employees of Brookhaven Science Associates, LLC under Contract No. DE-AC02-98CH10886 with the U.S. Department of Energy. The United States Government retains a nonexclusive, paid-up, irrevocable, world-wide license to publish or reproduce the published form of this document, or allow others to do so, for United States Government purposes. 


\title{
RHIC Low Energy Beam Loss Projections
}

\author{
T. Satogata
}

August 25, 2009

\section{Introduction}

For RHIC low-energy operations, we plan to collide Au beams with energies of $E=2.5-10 \mathrm{GeV} / \mathrm{u}$ in RHIC. Beams are injected into collision optics, and RHIC runs as a storage ring with no acceleration. At these low energies, observed beam lifetimes are minutes, with measured beam lifetimes of $3.5 \mathrm{~min}$ (fast) and $50 \mathrm{~min}$ (slow) at $\mathrm{E}=4.6 \mathrm{GeV} / \mathrm{u}$ in the March 2008 test run. With these lifetimes we can operate RHIC as a storage ring to produce reasonable integrated luminosity. This note estimates beam losses and collimator/dump energy deposition in normal injection modes of low energy operation.

The main question is whether a normal injection run is feasible for an FY10 10-15 week operations run from a radiation safety perspective. A peripheral question is whether continuous injection operations is feasible from a radiation safety perspective. In continuous injection mode, we fill both rings, then continuously extract and reinject the oldest bunches that have suffered the most beam loss to increase the overall integrated luminosity. We expect to gain a factor of 2-3 in integrated luminosity from continuous injection at lowest energies if implemented[1]. Continuous injection is feasible by FY11 from an engineering perspective given enough effort, but the required extra safety controls and hardware dose risk make it unappealing for the projected luminosity improvement. Low-energy electron cooling will reduce beam losses by at least an order of magnitude vs normal low-energy operations, but low energy cooling is only feasible in the FY13 timescale and therefore beyond the scope of this note.

For normal injection low energy estimates we assume the following:

- RHIC beam total energies are $\mathrm{E}=2.5-10 \mathrm{GeV} / \mathrm{u}$. (Continuous injection mode is probably unnecessary above total energies of $\mathrm{E}=7-8 \mathrm{GeV} / \mathrm{u}$.)

- RHIC operates only as a storage ring, with no acceleration.

- 110 bunches of about $0.5-1.0 \times 10^{9}$ initial bunch intensities (50-100\% injection efficiency, likely conservative)

- $90 \%$ collimation efficiency, with $10 \%$ of beam losses elsewhere. This is considered mildly conservative $[2]$.

- Beam lifetimes range from about 1 minute $(\mathrm{E}=2.5 \mathrm{GeV} / \mathrm{u})$ to 20 minutes $(\mathrm{E}=7-8 \mathrm{GeV} / \mathrm{u})$

- The primary beam loss mechanism is space charge/IBS blowup of transverse emittance. This implies that most beam is lost at transverse apertures, particularly abort kickers and collimators. Mar 112008 test run data also shows some mild chronic losses around the other IR triplets. We were not using collimators during this test run. (See Fig. 1.)

- The RHIC ASE states that the "maximum number of heavy ions in each ring (is) to be less than the equivalent of $2.4 \times 10^{11} \mathrm{Au}$ ions at $100 \mathrm{GeV} / \mathrm{u}$ " [3]. The total stored beam energy of this beam is $9.5 \times 10^{15} \mathrm{GeV}-\mathrm{u}$.

\section{Normal Operations (possible FY10 scenario)}

In this scenario we fill each RHIC ring, cog beams into collision, then dump and refill after 1-2 beam lifetimes. No modifications to the existing kickers are needed, and abort scenarios are the same as normal operations. 


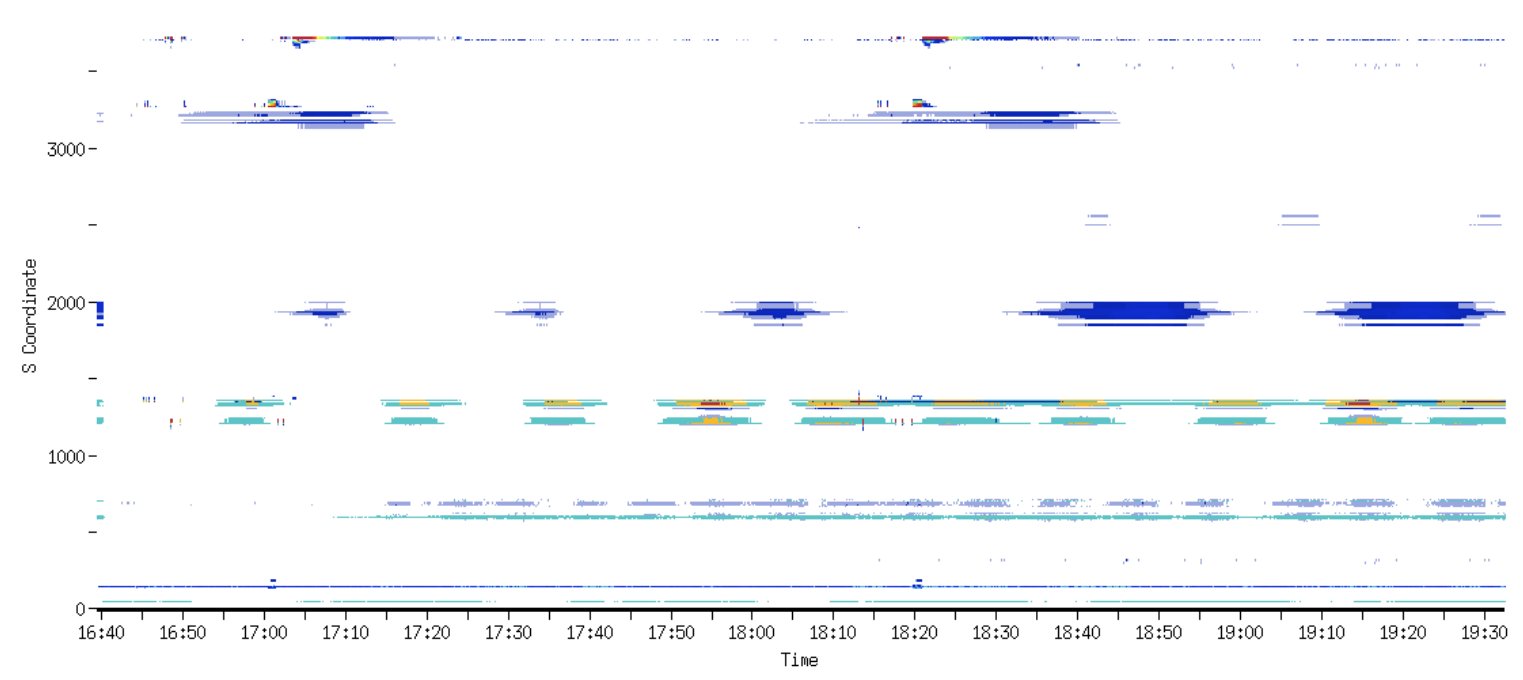

Figure 1: Beam losses during physics stores from fill 10005, Mar 112008 during E-4.6 GeV/u RHIC low energy tests. The vertical axis is s coordinate; chronic losses can be seen at IR6, IR8 (s=600m), IR12 $(\mathrm{s}=1800 \mathrm{~m})$, and IR4 $(\mathrm{s}=3200 \mathrm{~m})$. Large chronic losses are apparent at the abort kicker apertures in IR10 $(\mathrm{s}=1200 \mathrm{~m})$. Losses near $\mathrm{s}=3700 \mathrm{~m}$ at 17:05 and 18:25 are yellow injection losses. The times correspond to those of Fig. 2.

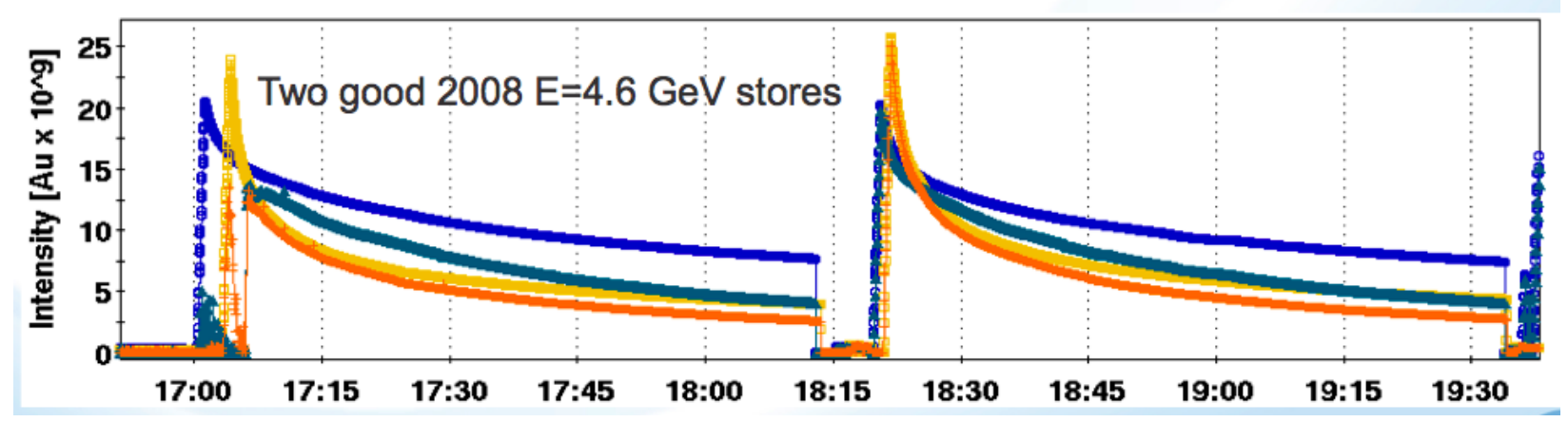

Figure 2: Two good beam physics stores from fill 10005, Mar 112008 during E=4.6 GeV/u RHIC low energy tests. Blue and yellow correspond to the two RHIC rings, and separate traces correspond to total and bunched beam intensities. The times correspond to those of Fig. 1.

This produces about 20-60 fills per day. Other projected beam parameters are listed in Table 1 for several energies of interest.

During the 2008 test, we had 1 hour stores that lost about $10^{10} \mathrm{Au}$ ions of total energy $E=4.6 \mathrm{GeV} / \mathrm{u}$ in each ring per hour in the reasonably-tuned blue ring. (See Fig. 2.) With these losses, total losses are about $1.8 \times 10^{13} \mathrm{GeV}-\mathrm{u} / \mathrm{hr}$.

The lowest energy desired FY10 scenario of $E=3.6 \mathrm{GeV} / \mathrm{u}$ raises this estimate by a factor of $4-10$, from increasing intensity and loss rates by factors of 2-3 each. This scenario then has $7.2-18 \times 10^{13} \mathrm{GeV}-\mathrm{u} / \mathrm{hr}$ total projected losses, or $7.2-18 \times 10^{12} \mathrm{GeV}-\mathrm{u} / \mathrm{hr}$ losses that escape collimation, and $6.5-16 \times 10^{13} \mathrm{GeV}-\mathrm{u} / \mathrm{hr}$ losses in the collimators. These ranges are consistent with estimates listed in Table 1.

The uncollimated loss rates are rather small. For example, $7.2-18 \times 10^{12} \mathrm{GeV}-\mathrm{u} / \mathrm{hr}$ integrated over 320-530 hours of operation is equal to the ASE stored beam limit of $9.5 \times 10^{15} \mathrm{GeV}$-u for a single loss event that stops operations for one hour.

Can the collimators handle the heat load? $10^{13} \mathrm{GeV} / \mathrm{hr}$ is 0.4 Watts, so this mode puts about 2.6-6.4 Watts of head load on the collimators, and one tenth of this is lost elsewhere. This is comfortably below the cryo heat load threshold of $1 \mathrm{~W} / \mathrm{m}$ even if the non-collimator losses are confined to a small region. The collimators are water-cooled copper blocks and should be able to comfortably dissipate this heat load [2]. 
Table 1: Nominal RHIC low energy operations chronic beam loss scenarios. Here we inject into each ring, then keep stored beams in collision before dumping both and refilling again. RHIC Au beam lifetime and luminosity lifetime have not been measured below $\sqrt{s_{N N}}=9.2 \mathrm{GeV}$.

\begin{tabular}{|l|c|c|c|c|c|c|}
\hline CM energy $\sqrt{s_{\mathrm{NN}}}[\mathrm{GeV}]$ & 5 & 7.7 & 8.3 & 9.2 & 11.5 & 18 \\
Beam energy E $[\mathrm{GeV}]$ & 2.5 & 3.85 & 4.15 & 4.6 & 5.75 & 9 \\
Fills/hr & 4 & 3 & 2 & 1 & 0.5 & 0.3 \\
\# bunches & 110 & 110 & 110 & 110 & 110 & 110 \\
AGS bunch intensity $\left[10^{9}\right]$ & 1 & 1 & 1 & 1 & 1 & 1 \\
Injection efficiency & 0.3 & 0.4 & 0.5 & 0.6 & 0.7 & 0.8 \\
Total RHIC intensity $\left[10^{9}\right]$ & 66 & 88 & 110 & 132 & 154 & 176 \\
\hline \hline ATR loss/fill $\left[10^{9}\right]$ & 154 & 132 & 110 & 88 & 66 & 44 \\
ATR loss/hr $\left[10^{9}\right]$ & 616 & 396 & 220 & 88 & 33 & 13.2 \\
ATR loss/hr $\left[10^{13} \mathrm{GeV}-\mathrm{n}\right]$ & 30.34 & 30.03 & 17.99 & 7.97 & 3.74 & 2.34 \\
\hline \hline RHIC loss/fill $\left[10^{9}\right]$ & 41.72 & 55.63 & 69.53 & 83.44 & 97.35 & 111.25 \\
RHIC loss/hr $\left[10^{9}\right]$ & 166.88 & 166.88 & 139.07 & 83.44 & 48.67 & 33.38 \\
RHIC loss/hr $\left[10^{13} \mathrm{GeV}-\mathrm{n}\right]$ & 8.22 & 12.66 & 11.37 & 7.56 & 5.51 & 5.92 \\
\hline Collimator efficiency & 0.9 & 0.9 & 0.9 & 0.9 & 0.9 & 0.9 \\
Collimator loss/hr $\left[10^{13} \mathrm{GeV}-\mathrm{n}\right]$ & 7.40 & 11.39 & 10.23 & 6.81 & 4.96 & 5.33 \\
<Collimator loss rate $>$ /ring $[\mathrm{W}]$ & 1.65 & 2.53 & 2.28 & 1.51 & 1.10 & 1.19 \\
\hline Excess loss/hr $\left[10^{12} \mathrm{GeV}\right.$-n] & 8.22 & 12.66 & 11.37 & 7.56 & 5.51 & 5.92 \\
<Excess loss rate $>[\mathrm{W}]$ & 0.37 & 0.56 & 0.51 & 0.34 & 0.25 & 0.26 \\
Excess loss/s $\left[10^{6} \mathrm{Au}\right]$ & 4.64 & 4.64 & 3.86 & 2.32 & 1.35 & 0.93 \\
\hline
\end{tabular}

Table 2: RHIC Run 10 (FY11) energies and operational times, per [4].

\begin{tabular}{|c|c|c|c|}
\hline $\begin{array}{l}\sqrt{s_{\mathrm{N} N}} \\
{[\mathrm{GeV}]}\end{array}$ & $\begin{array}{c}\text { Total Beam } \\
\text { Energy E } \\
{[\mathrm{GeV} / \mathrm{u}]}\end{array}$ & $\begin{array}{c}25 \text { week } \\
\text { scenario } \\
\text { [weeks] }\end{array}$ & $\begin{array}{c}30 \text { week } \\
\text { scenario } \\
\text { [weeks] }\end{array}$ \\
\hline 18 & 9 & 0 & 1.5 \\
\hline 11.5 & 5.75 & 1.5 & 2.5 \\
\hline 7.7 & 3.85 & 1.0 & 1.0 \\
\hline
\end{tabular}

\section{FY10 Scenarios}

The nominal RHIC run plan for Run 10 (FY11) contains options for 25- and 30-cryoweeks due to budget uncertainty. It mostly consists of operations above RHIC injection energy. There are, however, three energies below RHIC injection energy planned, as listed in Table 2[4]. Each operational time listed in this table includes some startup time, so physics conditions listed in Table 1 are appropriate for about $75-80 \%$ of the time listed, and very conservative during the remainder of the startup time that consists of single bunch injection with lower intensity bunches.

\section{References}

[1] E. Pozdeyev, private communication.

[2] A. Drees, private communication.

[3] C-AD Operations Procedures Manual 2.5.2, "RHIC Accelerator Safety Envelope Parameters", Section 5.1.1.

[4] S. Vigdor, "RHIC Run 10 Plan", RHIC News, Aug 11 2009, retrieved from web location http://www.bnl.gov/rhic/news/081109/story1.asp. 